1 A first simulation of soil-laser interaction investigation for soil

\section{characteristic analysis}

Karen M. Donaldson, Xiu T. Yan

Department of Design, Manufacture and Engineering Management, Space Mechatronic Systems Technology, Faculty of Engineering, Glasgow, G1 1XJ, UK

Laser Induced Breakdown Spectroscopy (LIBS) is an important technique utilised in several areas including that of agriculture and space exploration. However, whilst LIBS provides a new way of analyzing chemical composition of targeted soils or rocks, the quality and repeatability of the results are affected by the terrain and soil conditions as a result of physical matrix effects which occur due to varying properties like specific heat and thermal conductivity. These physical and chemical matrix effects cause difficulties with quantitative LIBS analysis. Together with this, the diverse areas in which LIBS is utilized means that it can require varying conditions of ablation techniques. Therefore, it is prudent to investigate theoretically the effect of different soil characteristics on the ablation process. The work presented here is the first simulation based research on soil quality analysis using LIBS. Aiming to gain insights into the soil breakdown process, laser coupling, sample temperature and its sensing performance through simulation of the laser ablation of soil using finite element modelling software. The proposed model within COMSOL Multiphysics was designed and developed to study the influence of multiple nanosecond (ns) laser pulses on the surface of samples of soil with varying properties. The simulation results reveal the simulated soil sensing behaviour for the first time. The 
28 computational results were compared to those obtained from LIBS experiments 29 conducted for the Argibot project at the University of Strathclyde.

30

31 


\section{INTRODUCTION}

33 Laser Induced Breakdown Spectroscopy (LIBS) is a technique based on the 34 emission spectroscopy of a material, which is caused by ablation of the sample 35 surface by heat from pulsed laser impact which creates a plasma plume. The 36 elemental composition is determined by the wavelengths and intensities of the 37 atomic emissions from the plasma. [Radziemski and Cremers, 2013; Anabitarte 38 et al., 2012; Hussain and Gondal, 2013]. Multiple LIBS analysis spots on a 39 single sample can provide information on individual elements contained within 40 the sample as well as providing a bulk composition. The LIBS technique is 41 sensitive to many key nutrients and elements in soil, and can allow for direct 42 observation of light elements such as H, C, N, O and B [Trevizan, 2008]. The quick detection of soil nutrient elements is of great benefit for crop yield and agricultural production [He, 2018]. The analysis of soil elements is also important for forensic soil discrimination, for creating a profile for soil types

46 with locations to compare with databases [Jantzi and Almirall, 2011].

47 One element of particular interest, as it is an essential element for plant growth, 48 is nitrogen [Sharma and Bali, 2018]. For this reason, the measurement of 49 nitrogen in soils is of specific interest in both agricultural and space science for 50 rich crop harvests and planetary habitation.

51 The advantage of using LIBS for elemental analysis is that the results are 52 available in real time, in-situ, and the measurements can be performed directly 53 on the sample surface without much, or any, sample preparation [Harmon et al 54 (a), 2005][Harmon et al (b), 2013]. The various areas in which LIBS can be 55 utilized means that it can have varying requirements and specifications of 
56 ablation techniques as the quality and repetition of the results can be affected by

57 the sample medium.

58 Due to its natural inhomogeneous characteristics soil, in particular, is a medium

59 that can produce varying and non-repeatable LIBS results. The application of

60 LIBS in agriculture is more challenging than other areas due to the complex

61 interactions between the sensors and samples. In agriculture the physical and 62 chemical characteristics of the samples can vary significantly in both space and

63 time. Thus, the parameters that are appropriate for other areas may not be

64 suitable for agricultural purpose [Peng, 2016]. Therefore, it is important to

65 investigate theoretically the effect of different soil characteristics on the ablation

66 process. The work presented here is the first simulation based research on soil

67 quality analysis using laser ablation, aiming to gain insights into the soil and

68 laser interaction through simulation of laser soil ablation using finite element

69 modelling software.

70 The work is part of a project at the University of Strathclyde named Agribot, 71 which is an autonomous ground rover platform for monitoring and intervention 72 tasks on modern farms and space exploration [Post et al., 2017]. Part of the 73 rover's ability is to conduct soil analysis through a mounted portable LIBS 74 system.

75 For a typical LIBS system, nanosecond (ns) or femtosecond (fs) laser pulse 76 durations are used. The laser pulse length can dramatically change the physics of 77 the laser-induced plasma creation. Those from fs laser pulses and ns pulses can 78 differ considerably. The difference is due to how the laser pulse interacts with 79 the sample. A fs laser pulse interacts only with the electron subsystem, whilst ns 80 laser pulses continuously interact with different thermodynamic states of the 
81 material, starting from solid, through to a plasma. These differences are observed,

82 also, in the area and shape of the ablation crater left on the samples surface

83 [Freeman, 2013].

84 It has been recognised that short laser pulses and UV wavelengths largely 85 provide the best performance metrics concerning precision, accuracy and 86 sensitivity when laser sampling is coupled with inductively coupled plasma (ICP) 87 spectroscopy for quantitative analysis [Yang, 2009].

88 The properties of LIBS plasmas and therefore sample analysis depend on the 89 operational parameters of the system, including; the nature of the laser pulse 90 such as the energy, duration, repetition rate, and number of laser shots [Hahn and 91 Omenetto, 2012]. The properties of the sample [Rauschenbach et al, 2008] and 92 the ambient atmosphere are also a factor. LIBS measurements are also sensitive 93 to environmental factors, such as the chemical and physical properties of the 94 sample and the homogeneity and ambient states of the sample. For example, the 95 air temperature and pressure. The particle size of the sample can also affect both 96 the LIBS measurements along with how the laser power interacts with the 97 sample [Gustinelli, 2015].

98 To investigate parameters for achieving optimal results from the ablation process, 99 it is necessary to employ a simulation method that can effectively reflect the 100 absorption coefficient, conduction length and pulse energy for various types of 101 samples.

102 The present study simulates ns laser ablation using heat conduction theory in a 103 two-dimensional COMSOL model to investigate the interaction between the 104 laser pulse and various soil conditions.

\section{LASER MACHINE INTERACTION}

107 Ablation by intense laser pulses is widely used for ionization of samples.

108 However, the LIBS technique can have low sensitivity in trace elements 109 detection which is commonly related to the small quantity of ablated material in 
110 micro-analyses. The texture and surface of, in particular, a soil sample can have

111 a large effect on how much soil is ablated in the process.

112 Due to heat conduction, when a high intensity laser beam impacts the surface of

113 a material, the surface temperature quickly rises. This in turn causes the surface,

114 that has risen to the ablation temperature, to vaporize and create a plasma

115 containing molecules of various elements enclosed within the sample. The

116 temperature of the sample will rise and fall due to its heating and cooling before

117 and after a laser pulse respectively.

118 For ns lasers, much of the latter part of the energy pulse is used in heating the

119 plasma formed during the earlier part of the pulse through linear absorption. This

120 results in a large area of the sample being affected by the heat. Any plasma that

121 is formed is extremely ionized which causes continuum emission. Gated

122 detectors allow for a reduction in background noise and thus improvement the

123 signal-to-noise ratio while minimizing any spectral interferences between species.

124 Soil is naturally chemically and physically a complex matrix with differing

125 chemical compositions and physical properties [Jantzi and Almirall, 2011].

126 Physical matrix effects, which are more complex and difficult to perfect than

127 chemical ones, occur during the ablation step of the LIBS process because of

128 material differences in such properties as the thermal conductivity, specific heat,

129 and latent heat of vaporization. Also, the characteristics of a sample such as grain

130 size, hardness, coherence, and moisture contents. There has been research

131 conducted by Carvalho et al to understand the influence of the particle size

132 distribution on the matrix effect in pellets of plant materials [Carvalho et al.,

133 2017]. Other factors that produce any variability on the surface texture will affect

134 the degree of laser energy coupling, the laser pulse energy distribution within the 
135 material, and therefore the performance of a LIBS system and the degree of

136 material ablation. The mass of material ablated is another matrix effect that can

137 change the signal intensity for a particular element between samples with the

138 same concentration [Hahn and Omenetto, 2012].

139 Thus, these physical matrix effects make quantitative LIBS analysis much more

140 problematic. Research has been conducted by Bousquet et al for quantitative

141 LIBS on soil samples for clay and sand type soils which demonstrated that LIBS

142 signals for soil samples are strongly affected by the matrix effects [Bousquet et

143 al., 2007]. Currently, for quantitative LIBS it is important to have standards that

144 are very closely matrix matched to the type of samples being analyzed which

145 will allow for excellent accuracy and precision [Jantzi and Almirall, 2011].

\section{III SOIL HEAT TRANSFER PROPERTIES}

148 The term 'soil', as used by engineers, refers to a complex material consisting of

149 solid particles of various compositions (mineral and/or organic) and of various

150 shapes and sizes that are randomly arranged with pore spaces between them.

151 Soils are composed of different ingredients for example sand and clay. Clayey

152 soils are heavy, high in nutrients, wet and cold in winter and baked dry in

153 summer and sandy soils are light, dry, warm, low in nutrients and often acidic.

154 The thermal conductivity of a soil is defined as the amount of heat passing in

155 unit time through a unit cross sectional area of the soil under a unit temperature

156 gradient applied in the direction of this heat flow. It must be assumed that the

157 sample of soil is much larger in comparison with a representative cell of a

158 homogenous soil. In order to create samples within the simulation that replicate

159 similar properties of the experimental setup, four different soil samples with 
160 varying properties were investigated. The main properties that differentiated

161 them were the thermal conduction, porosity, specific heat, density and water

162 content. The texture and composition of a soil can vary due to several factors;

163 these main factors can be divided into three categories.

\section{A. Moisture}

166 The composition of naturally occurring soil varies continuously, mainly from the

167 continuously varying temperature field to which the soil is subject. These

168 changing temperature gradients alter the soil composition, particularly in the

169 amount, phase and condition of the water which in-turn leads to variations in the

170 thermal properties of the soil. Geomaterial thermal properties, such as thermal

171 conductivity, vary depending on the type of geomaterial used and the degree of

172 saturation. For soils, fine dry sand has low thermal conductivity values and silty

173 sandy gravel has high thermal conductivity values. The thermal conductivity for

174 these materials can range between $0.15 \mathrm{~W} / \mathrm{mK}$ and $4.4 \mathrm{~W} / \mathrm{mK}$ respectively.

B. Porosity

177 Porsity (void fraction) is a measure of the void spaces in a material, and is a

178 fraction of the volume of voids over the total volume. These values range

179 between 0 and 1 , or as a percentage between 0 and $100 \%$. The porosity of soils

180 can vary widely, for example the porosity of loose soils can be about $n=50 \%$,

181 whilst the porosity of compact soils is about $n=30 \%$. The value of the porosity

182 depends on grain size distribution; it follows that the higher the porosity value

183 the smoother the grain size distribution. 


\section{Grain size}

186 The grain size distribution of a soil implies a certain pore size distribution which

187 determines the permeability of the soil. Expressed as the permeability, K, as a

188 function of the soil porosity $\mathrm{n}$ and its internal surface area per unit volume $\mathrm{S}$ is

189 given by the equation $\mathrm{K}=\mathrm{Dn}(\mathrm{n} / \mathrm{S})^{2}$ in which $\mathrm{D}$ is a constant, dependent on the

190 viscosity of the water. As the grain size decreases, the specific surface area 191 increases rapidly, leading to a substantial decrease in the permeability and an

192 increase in the number of capillaries per unit volume.

\section{IV. AGRIBOT EXPERIMENTS}

195 As previously mentioned these simulations were conducted in parallel with experimental LIBS work for the Agribot project. The Agribot rover is adapted to

197 support a portable LIBS system. The system consists of a laser, a sample dish

198 within a chamber and spectrometers which are connected to a computer system

199 for data collecting and processing. The laser is a Nd:YAG operating at $1064 \mathrm{~nm}$

200 with a beam diameter of $3 \mathrm{~mm}$, and a repetition rate of $1 \mathrm{~Hz}$, the pulse energy of $20125 \mathrm{~mJ}$ and a nominal pulse width of $4 \mathrm{~ns}$.

202 The soil was collected from agricultural land in central Scotland from differing 203 locations around the field. The final samples prepared for the LIBS experiments

204 varied in grain size and porosity. Throughout the experiments variations in the 205 depth, width and shape of the ablation holes of the samples were noted.

206 The soil underwent several pre-treatment regimes to prepare for testing. Three

207 different soil samples were prepared. The field moist soil, initially, was baked at $20840^{\circ} \mathrm{C}$ for two days to dry it and then passed through a $1 \mathrm{~mm}$ sieve. Following 209 from this the soil was milled, to $<100 \mu \mathrm{m}$, using a planetary ball mill. Finally, it 
210 was pressed into a pellet, without the use of any binding agent, using a manual

211 laboratory press $(\mathrm{P}=7 \mathrm{t})$ for 30 seconds.

212 It was observed that the different textures of soil samples ablated in a variety of

213 ways after 10 laser pulses, confirming how the physical matrix effect can cause

214 ablation craters to change in shape and dimensions. It was noted that there was

215 also a reduction in spectrum noise level with a decrease in particle size.

216 The laser pulse effect on the dried loose sample created a relatively large area of

217 depression compared to that of the milled, pelleted soil, Fig 1. There was an

218 observable area of depression after 10 laser pulses and the quality of the hole

219 was not high. Much of the soil around the ablation area had also been moved and

220 distorted.

221 The milled soil displayed an ablation hole that was significantly smaller than that

222 of the dried loose soil and there appeared to be no ridged edge, Fig. 1. In general,

223 it was found that an increase in particle size contributed to increases in the

224 diameter of the crater. There was also an increase in the abundance and size of

225 fractures forming off the ablated crater edge and hence, the edge smoothness of

226 the ablated crater was also found to be proportional to the particle size.

227 The elemental spectrum waveform obtained during the LIBS experiments, for

228 different soil samples, were observed for variations. As nitrogen is available in

229 different forms, the specific wavelength for the form of interest must be observed

230 in the spectral results for analysis. It has been found, from previous works, that

231 gaseous nitrogen can be resolved at several wavelengths between $\sim 400 \mathrm{~nm}$ and

$232600 \mathrm{~nm}$ [Hanafi et al., 2000] and nitrogen species within soil matrix are resolved

233 at a wavelength of $\sim 746 \mathrm{~nm}$ [Dong et al., 2013][Harris et al., 2004]. 
234 In general, it was discovered that the intensities of nitrogen and other elements 235 varied shot to shot which introduced the need to average the results obtained

236 from several laser pulse shots onto the sample's surface. This is due to the nature

237 of soil, as it is regularly inhomogeneous and contains pockets of elements in

238 varying quantities.

239 The spectrum obtained from the sample of dry loose soil was the most 240 susceptible to exhibiting this nature of large variations in the levels of nitrogen

241 gained between laser shots. There were improvements in the noise of the spectra

242 and the varying levels of element concentrations when the sample of the soil was

243 milled and pelletized. The spectrum noise and the variations in the levels of 244 nitrogen between pulses was less varied for milled soil.

246 V.SIMULATIONS

\section{A. Model development}

248 The dimensions and boundary for the soil samples are as described in Fig. 2 and

249 Fig. 3. As this research is a continuation from the Argibot analysis experiments 250 the simulations have been modelled to replicate the key properties of samples 251 used in the analytical experiments.

252 Whilst the modelled samples do not replicate the exact property values of the

253 field soil samples, they have been modelled to replicate the variations in porosity 254 and, for scientific comparison, dry density and moisture levels. In the present 255 study, the sample was simplified to a 2D rectangle to solve for the boundary of 256 the laser heated segment i.e. the area of the sample that is not irradiated can be

257 disregarded. Therefore, the modelled geometry is much smaller compared to that 258 of the $\sim 3 \mathrm{~mm}^{2}$ soil sample used in the experiments, Fig 2. 
259 It is possible from this $2 \mathrm{D}$ model to observe the varying depth and width of the

260 ablated areas caused by the interactions with the laser pulse for varying sample

261 specifications.

262 The properties of the laser are presented in Table 1, taken from the portable

263 LIBS system specifications. A laser beam of diameter $3 \mathrm{~mm}$ FWHM hits the

264 surface of the soil sample of width and thickness of $1 \mathrm{~mm}$ and $20 \mu \mathrm{m}$

265 respectively. The laser is modelled as a heat source with Gaussian distribution,

266 defined by the equation shown in Table 2.

267 The ablation is modelled as an ablative heat flux, the parameters set for the

268 ablation conditions are shown in Table 1. As the material is removed from

269 interaction with the laser pulse energy, a model of the material removal must be

270 applied. This is calculated through the value for the heat of sublimation to

271 calculate the ablation velocity i.e. the rate at which the material is removed,

272 Table 2.

273

274 B. Boundary conditions

275 Two types of physics solution, namely laser heat transfer and geometry 276 deformation are employed to simulate the mechanism of laser induced

277 breakdown. For each of these areas, appropriate boundary conditions have to be

278 specified before the problem can be solved. The boundary conditions for the

279 material are shown in Fig. 4, they are mapped such that the material removing

280 process can be resolved and the side and bottom boundaries of the sample are

281 assumed to be thermally insulated. Due to this, the convection effect is only

282 considered on the top surface. 


\section{Meshing conditions}

285 The heat generated from the laser source affects the surface of the sample as well

286 as the interior depending on the material absorption coefficient. The meshing

287 algorithm must be able to resolve this internal heat. A suitable resolved meshing

288 scale was calculated from the soil thermal diffusivity and the conduction length.

289 These parameters and corresponding equations are shown in Table 1 and Table 2

290 respectively. Thus, the simulated ablated area of the experimental sample is

291 solved with the affected area width $1 \mathrm{~mm}$, height $20 \mu \mathrm{m}$. The finite element

292 distribution and the maximum mesh size must be small enough compared with

293 the size of the laser irradiation region to resolve the heat transfer conduction

294 length of the laser.

295 Due to the length of the laser pulse, the time resolution is in the order of ns and

296 the laser absorption area has a maximum element size in the magnitude of $0.1 \mathrm{~nm}$.

297 The mapping of the mesh sizing is illustrated in Fig. 5.

\section{D. Simulation parameters}

300 The laser parameters were taken from those of the, previously described,

301 portable LIBS system. In order to investigate the material removal in-between

302 pulses a laser beam heat source was modelled with multiple laser pulses. In the

303 present work ten pulses over a total time duration of $2 \mu$ s (the first laser pulse

304 laser switches on at $0.1 \mu \mathrm{s}$ and remains on until $0.104 \mu \mathrm{s}$ ) were simulated, Fig. 6

305 illustrates the model of the simulated laser pulses.

306 The laser ablation is affected, and can be controlled by several process variables

307 namely laser pulse length and the soil texture i.e. grain size or moisture level.

308 Therefore, several variables and constants that were necessary for the simulation 
309 were globally parameterized. These were for the shape and width of the pulse

310 and material parameters such as specific heat capacity and thermal conductivity.

311 Table 1 summarises the properties of the soil and the laser ablation parameters

312 used in the computational model. In total four soil samples were modelled to

313 replicate the characteristics of the experimental samples namely, dry loose fine

314 sandy soil (dry), wet loose fine loose soil (saturated), fine sandy milled and

315 compressed soil (pelleted) and lastly for comparison; soft grey clay soil.

316

317 E. Governing Equations

318 In the present model, the heat transfer in the sample is conjugate heat transfer.

319 As the heat in the sample is due to conduction then it can be described by

320 Fourier's Law and as the sample analysis here is time dependent then we must

321 consider this. The heat source is distributed in time by a Gaussian distribution,

322 Fig. 7.

323 The governing equations associated with the boundaries employed in the heat

324 transfer model are also summarized in Table 2.

325 The addition of the deformed geometry function is used due to the changing 326 shape of the domain boundary caused by the laser ablation vaporising the surface

327 of the sample. The domain change is significant, so the deformed geometry 328 interface used was a hyperelastic smoothing type to deform the mesh. These 329 were, namely, controlled by the total heat flux experienced by the sample and the 330 ablation velocity associated with this. 


\section{SIMULATION RESULTS}

\section{A. Laser pulse analysis}

336 Illustrated in Fig. 8 is an example of the heating process effect on the soil sample

337 before, during and after one laser pulse.

338 It is seen that the laser pulse heats the entire surface of the modelled sample and

339 then cools from the middle to the edges. As the laser pulse hits the soil sample it

340 quickly transfers heat to whole surface. The area closest to the center sees a

341 temperature rise sufficient for ablation and the material is removed. It is seen that

342 in the centre of the pulse the laser power is at the highest and so the ablation and

343 material removal rate is the highest. Thus the material is removed faster than the

344 time it would take to conduct heat into the remaining sample. So when the laser

345 is in the OFF state the remaining material is not at as high temperature as directly

346 below the laser beam.

347 For sandy and sandy-clay samples the thermal conductivity and diffusivity

348 increase with the increase of moisture content. This property is evident in Fig. 9

349 where the difference in surface temperature is seen $3 \mathrm{~ns}$ after one laser pulse, for

350 the dry loose soil sample and clay soil sample. In the case where samples may

351 change in dry density, this increase in dry density will cause an increase in the

352 thermal conductivity.

353 B. Temperature

354 When the material temperature exceeds the ablation temperature it is assumed 355 that the material evaporates and elements contained within the sample are 356 removed. Fig. 10 shows the temperature distribution for all the soil samples after

357 each laser pulse. As silicates account for the largest percentage of the 358 composition of soil, the ablation value of $2000 \mathrm{~K}$, Table 1 , was chosen as an 
359 average value for heat of sublimation for the various silicates (along with some

360 organic materials) which should well represent the soil.

361 In comparison to ablation temperature, the maximum temperature after each

362 pulse is $\sim 2000 \mathrm{~K}$ which would suggest that there is ablation of the material after

363 each pulse. To compare, in more detail, the surface temperature for each soil

364 sample one pulse was analysed as shown in Fig. 11.

365 The temperature gradients illustrated in Fig. 10 and Fig. 11 illustrates a steep

366 change in temperature due to the rapid increase and decrease of the surface

367 temperature as the material experiences higher heating and cooling rates during

368 the laser ablation process.

369 It was observed that the temperature gradients change for each soil sample, Fig.

370 11. There have been various correlations made between the thermal conductivity

371 of soils and their density or porosity. An increase in the dry density of a soil,

372 leads to an increase in its thermal conductivity. This occurs as an increase in dry

373 density will mean there is more solid matter per unit soil volume and less pore

374 air/water per unit soil volume which allows for better heat transfer across the

375 contacts. This behaviour is exhibited by the dry milled soil; it can be seen that

376 the temperature reduces much quicker than the other soil samples.

377 In the instance of clay minerals, they have a thermal conductivity around four

378 times higher than of that of water. Sandy soils often contain quartz which has a

379 thermal conductivity about 15 times higher than water, which means that they

380 are expected to show a greater rate of increase in thermal conductivity with dry

381 density than clay soils. This is illustrated in the simulation when the rise and fall

382 of the temperature for wet loose and clay soil was investigated. 

that the solid fraction replaces some of the water pores. In this situation, for an increase in thermal conductivity the soil must have a higher thermal conductivity than the water it replaces.

\section{Ablation effects on sample surface}

389 The optical emission spectroscopy of the plasma that is produced by the laser390 matter interaction is the fundamental basis of LIBS. Hence, the efficiency of the

391 analytical performance is related to the laser-matter interaction.

392 Using the simulation model described previously, a parameter study of the

393 ablation process was performed. The ablation of the soil sample is affected and 394 controlled by several variables and therefore changes in the samples can affect 395 the efficiency of the ablation process.

396 Due to the Gaussian energy distribution the laser energy, and ultimately the 397 sample temperature, is highest at the centre of the beam, reducing towards the 398 edges of the pulse which creates an ablation area that has greater depth than 399 width [Gaudiuso et al., 2010]. Furthermore, the depth of the ablation area 400 increases with each additional laser pulse. Fig. 12 and Fig. 13 illustrate the 401 differences in ablation shape for two different types of samples.

402 In general, it can be seen that the sample of clay has ablated less than dry milled 403 fine soil. The change in gradient of the surface topology of the fine sandy clay 404 sample, Fig 12, is more distinctive than that of the dry milled sample, Fig 13.

405 Considering the, initial, sample height was $20 \mu \mathrm{m}$ it is possible to analyse how 406 the laser pulse energy changes the shape and size of the varying samples. The 
407 clay soil sample surface depletes from an initial $20 \mu \mathrm{m}$ to $18 \mu \mathrm{m}$ whilst the dry 408 milled sample depletes to a final height of $\sim 16 \mu \mathrm{m}$.

409 The density of the samples will affect the total volume of material ablated and

410 therefore the area of depression left behind, Fig. 14 illustrates this.

411 Referring to Fig. 14, as seen in the experimental results, the area of material

412 removed for the dry loose sample was greater than the milled sample. As 413 previously explained, due to the quartz content in sandy soils they should 414 demonstrate a greater rate of increase in thermal conductivity than clay soils.

415 A point evaluation was introduced to examine how the height of the pellet 416 changed with after each laser pulse. The point of evaluation is illustrated in Fig. 417 15, denoted by the red dot. Referring to Fig. 15, it is seen that as time progresses, 418 i.e. as more laser pulses are fired onto the sample surface, the dry loose sandy 419 soil decreases in height much more quickly than the others, especially to that of 420 the clay sample and the sample with added moisture.

421 It was noted that all the samples initially ablated at a similar rate until, 422 approximately, after three pulses of laser energy when they begin to ablate at 423 their own, different, rates. The sample that changed the most, and at the quickest 424 rate, was that of the dry, loose soil. This behaviour was exhibited in the 425 experiments. Referring to Fig 1, the dry loose soil in the sample container left the 426 largest diameter of depletion compared to that of the dry milled soil, this 427 behaviour is also exhibited in Fig 15. It was observed that a reduction in particle 428 size was related to a decrease in the diameter of the ablation crater

429 Finally, Fig. 16 illustrates the volume of material removed after ten shots of laser 430 energy. This is inclusive of the total volume of material removed from the 431 sample and not only the radius of the ablation area. Here, the effect that moisture 
432 has on the ablation process of the sample is obvious by comparing the volume

433 difference of the wet and dry loose soil.

434

\section{VII. Discussions and Conclusions}

436 Work here presented is aimed to investigate and report the effect a ns NG:YAG

437 laser, by analysis of ablation area and surface temperature, has on varying 438 samples of soil.

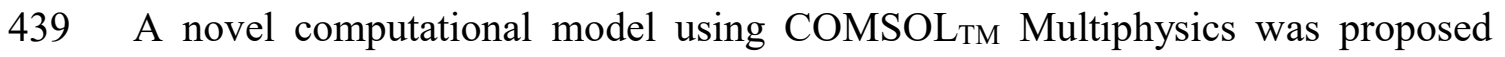
440 and developed to understand the influence of multiple laser pulses on the surface

441 of sample of soil for various soil characteristics. The investigation was based on

442 the assumption that the process occurs due to the material evaporation by heating

443 of ns laser pulses. The software is used to simulate this material removal during

444 the laser ablation process.

445 This research provides simulation details, a brief review and insight into soil446 laser interactions, for coupling and ablation, for various soil types and soil 447 properties. Details as such are significant knowledge as the physical matrix 448 effect can be considered a crucial aspect for quantitative LIBS analysis [Capitelli 449 et al., 2002].

450 Referring to the LIBS experimental findings, in general, it was found that an 451 increase in particle size contributed to increases in the diameter of the crater. 452 Additionally, it was found that there was a correlation between the sample type, 453 thus ablation, to the obtained experimental results primarily in the spectral noise 454 level. The simulation results exhibited ablation crater characteristics comparable 455 to that of previous LIBS experimental results. This work can help to predict laser 456 ablation of different types of soil and also gives insight into how the laser 
457 interacts with the soil samples. As discussed in the introduction, the work of

458 Gabriel Gustinelli et al. and Ines Rauschenbach et al. show there is a relation

459 between moisture, particle size of the sample which can affect both the LIBS

460 measurements and how the laser power interacts with the sample. This work

461 illustrates the interaction of the laser to the sample for possible explanation to the

462 experiments conducted in these papers and those similar. The modelled samples

463 of the wet loose soil illustrated the effect that moisture can have on the ablation

464 of a sample during laser induced breakdown. The differences between the wet

465 and dry soil samples, due to their differing water contents, is evident by the

466 volume of the ablation creator and temperature dissipation after the laser pulse

467 train impact.

468 These results are important when considering LIBS for different terrains whether

469 they be dry/saturated or with/without void. The model can also be adapted for

470 other laser specifications and samples. Simulation results such as these can be

471 used to predict, or verify, the effectiveness of the ablation process (quantity of

472 material removed) on various terrains and therefore the quality of the LIBS

473 analysis. This is applicable in both agricultural circumstance where terrain can

474 be water logged and clay-like or towards dry and sandy or in space exploration

475 [Knight et al., 2000] where, for example, Mars can vary between fine sediment

476 or large rocky terrains [Lazic et al., 2007]. This work can go towards providing

477 further research to understanding of the physical matrix effects of, in particular,

478 soil towards improved and repeatable LIBS spectrum and data for quantitative

479 analysis. It is also possible to create soil type profile databases for referencing

480 and to assist in producing matrix matching calibration charts. 
481 This research can be utilised in more wide-ranging areas such as to help 482 understand how soil heat flux is affected by porosity and moisture content. Such 483 information is important in agricultural systems and matters relating to climatic 484 changes [Castell et al., 1999; Han et al., 2013; Kondo and Saigusa, 1994; Sauer, $4852005]$.

486

487 Acknowledgement

488 This work was supported by the UK Space Agency. The authors would also like 489 to thank COMSOL for their help and advice.

490 
493 Fig. 1 Soil samples from experimental LIBS analysis for (a) dried and sieved soil,

494 (b) milled to $<100 \mu \mathrm{m}$ soil and (c) milled \& pelleted soil.

495

496 Fig. 2 Schematic of the soil sample pellet illustrating selected area for COMSOL

497 modelled laser ablation.

498

499 Fig. 3 Schematic of the soil sample pellet, illustrating dimensions of selected 500 area for COMSOL model.

501

502 Fig. 4 Modelling for simulation, illustrating the boundary conditions.

503

504 Fig. 5 Meshing condition for the sample model, illustrating refined meshing to

505 resolve area around ablation required for accurate simulated heat conduction.

506

507 Fig. 6 Modelled laser pulses.

508

509 Fig. 7 Gaussian distribution of laser intensity from the surface.

510

511 Fig. 8 Illustration of effect of the laser pulses on the surface temperature of the

512 sample (a) before any pulse impact $(0 \mu \mathrm{s})$, (b) during pulse heating $(0.101 \mu \mathrm{s})$

513 and (c) during cool down (pulse off - $0.105 \mu \mathrm{s}$ ). 
515 Fig. 9 Illustration of effect of the laser pulse on the surface temperature of (a)

516 sample of dry loose and (b) sample of clay at same time point $(0.107 \mu \mathrm{s})$

517

518 Fig. 10 Sample temperature with each pulse of the laser for all soil samples

519 recorded at the center of the sample.

520

521 Fig. 11 Example of sample temperature after five pulses of the laser for all soil

522 samples, illustrating when the pulse is on and off.

523

524 Fig. 12 Effect of the ablation process on a sample of clay. Illustrating the volume 525 of soil that has been removed.

526

527 Fig. 13 Effect of the ablation process on a sample of fine sandy dry milled and 528 compressed soil. Illustrating the volume of soil that has been removed.

529

530 Fig. 14 Shape and size of ablation sites for soil sample after ten pulses.

531

532 Fig. 15 Change in height of soil sample due to ablation after each laser pulse.

533

534 Fig. 16 Volume difference of each soil sample after ten pulses.

535

536

537

538

539 
(a)

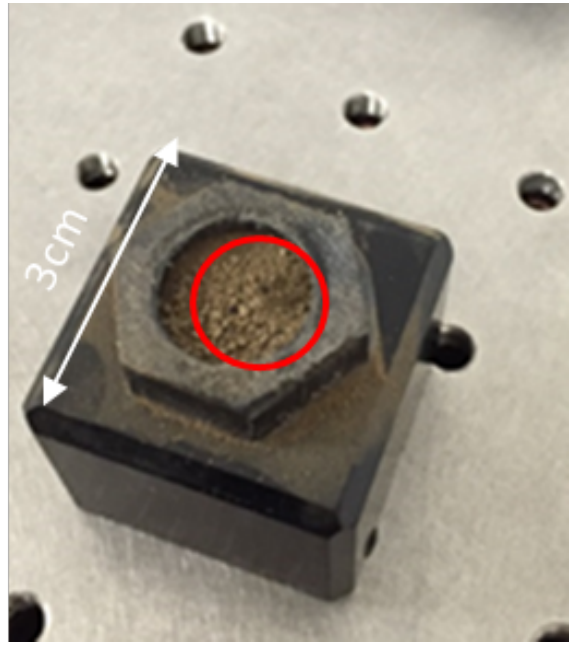

541

(b)

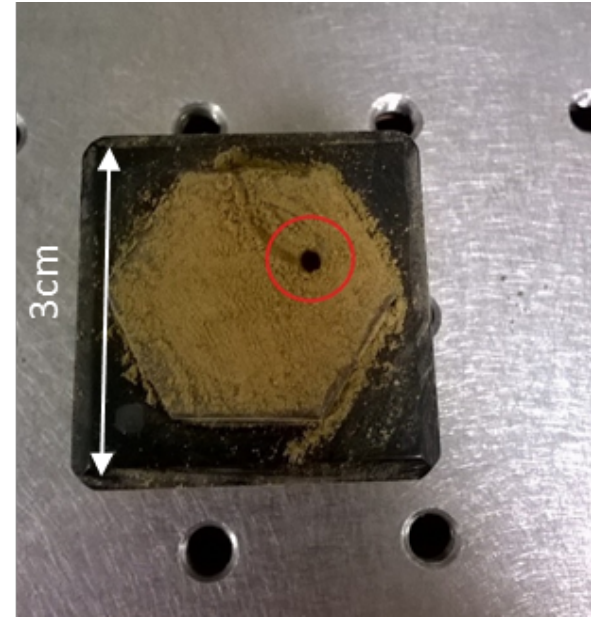

(c)

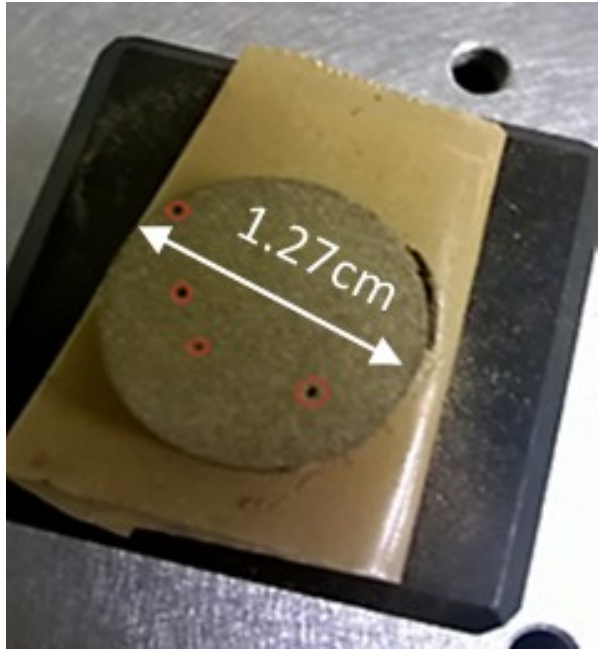

543

Fig. 1 


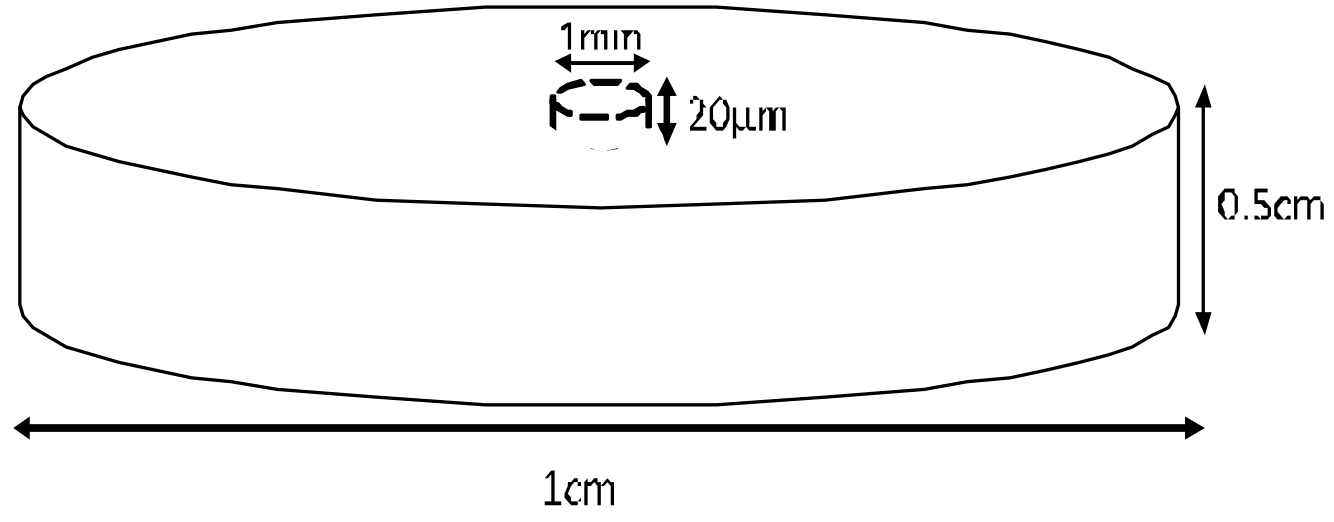

548

549

550

Fig. 2

551

552

553

554

555

556

557

558

559

560

561

562

563

564

565

566 


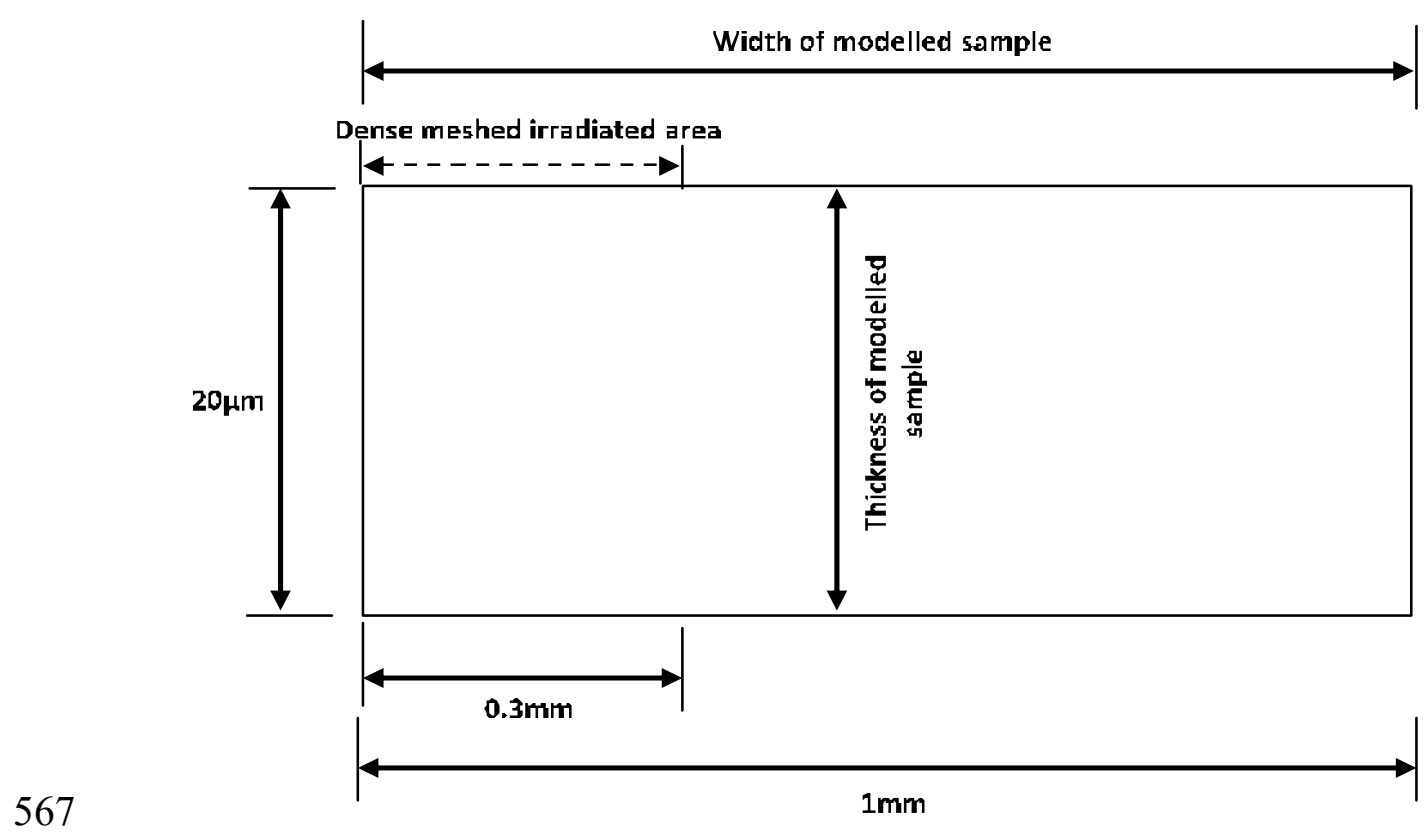

568

569

570

Fig. 3

571

572

573

574

575

576

577

578

579

580 


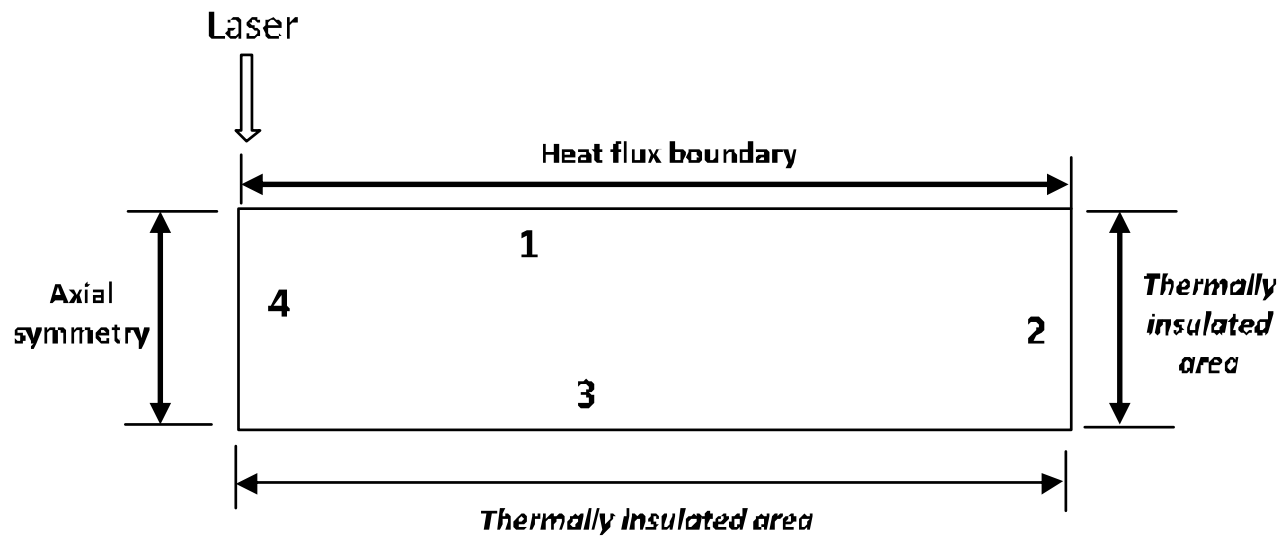

582

583

584

585

Fig. 4

586 


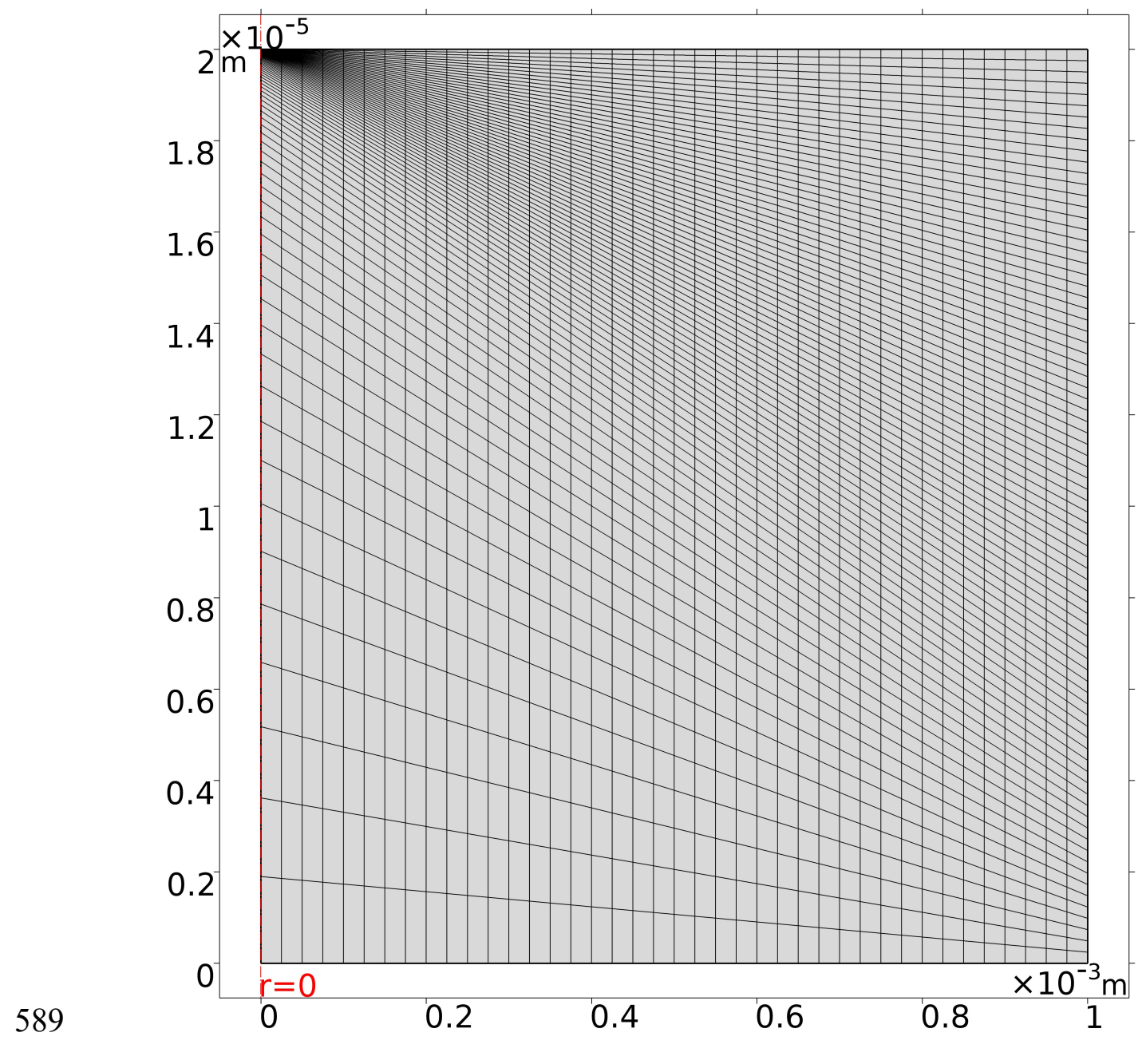

590

591

Fig. 5

592 
593

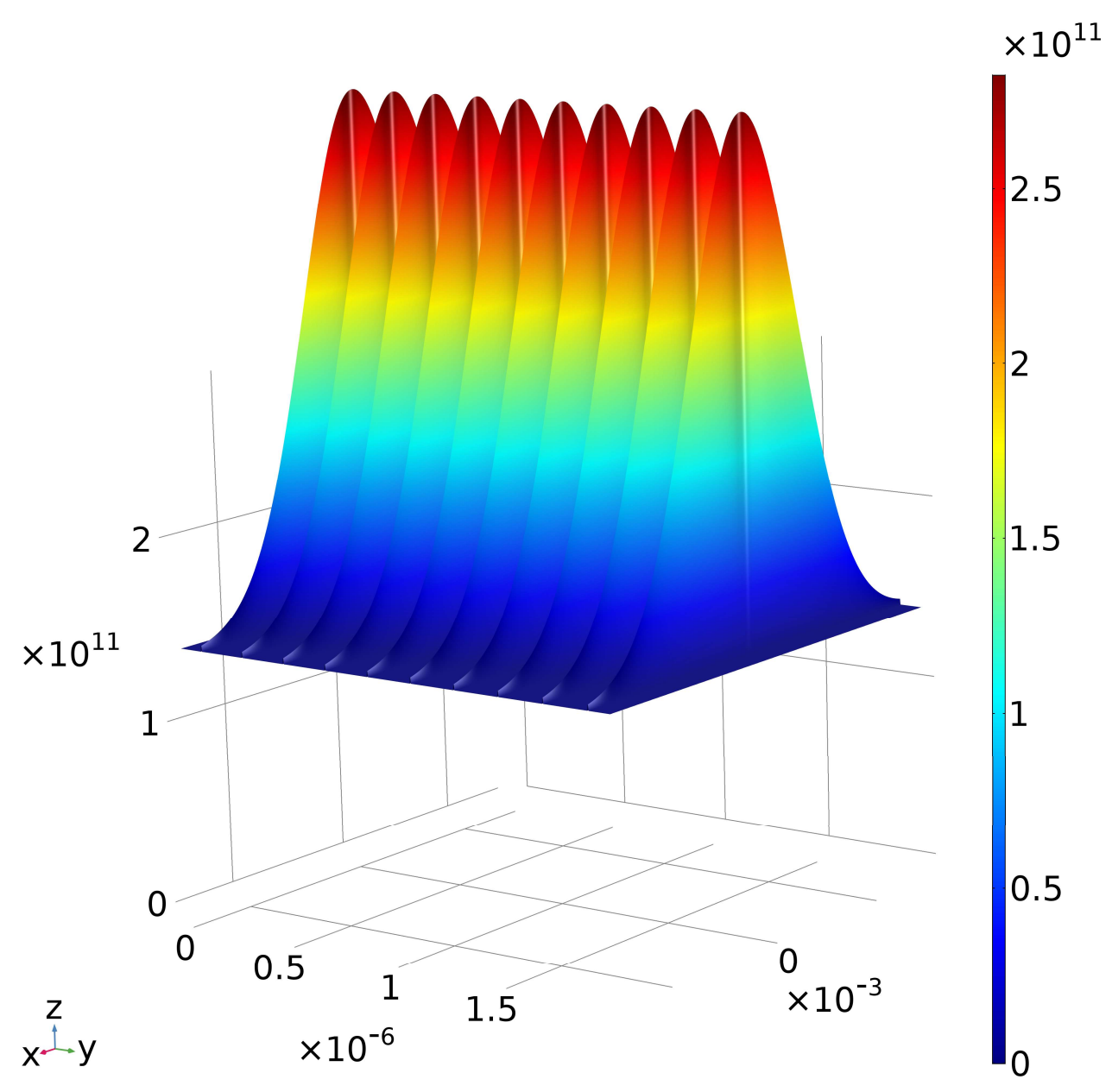

594

595

Fig. 6

596

597

598

599

600

601

602

603

604 
605

606

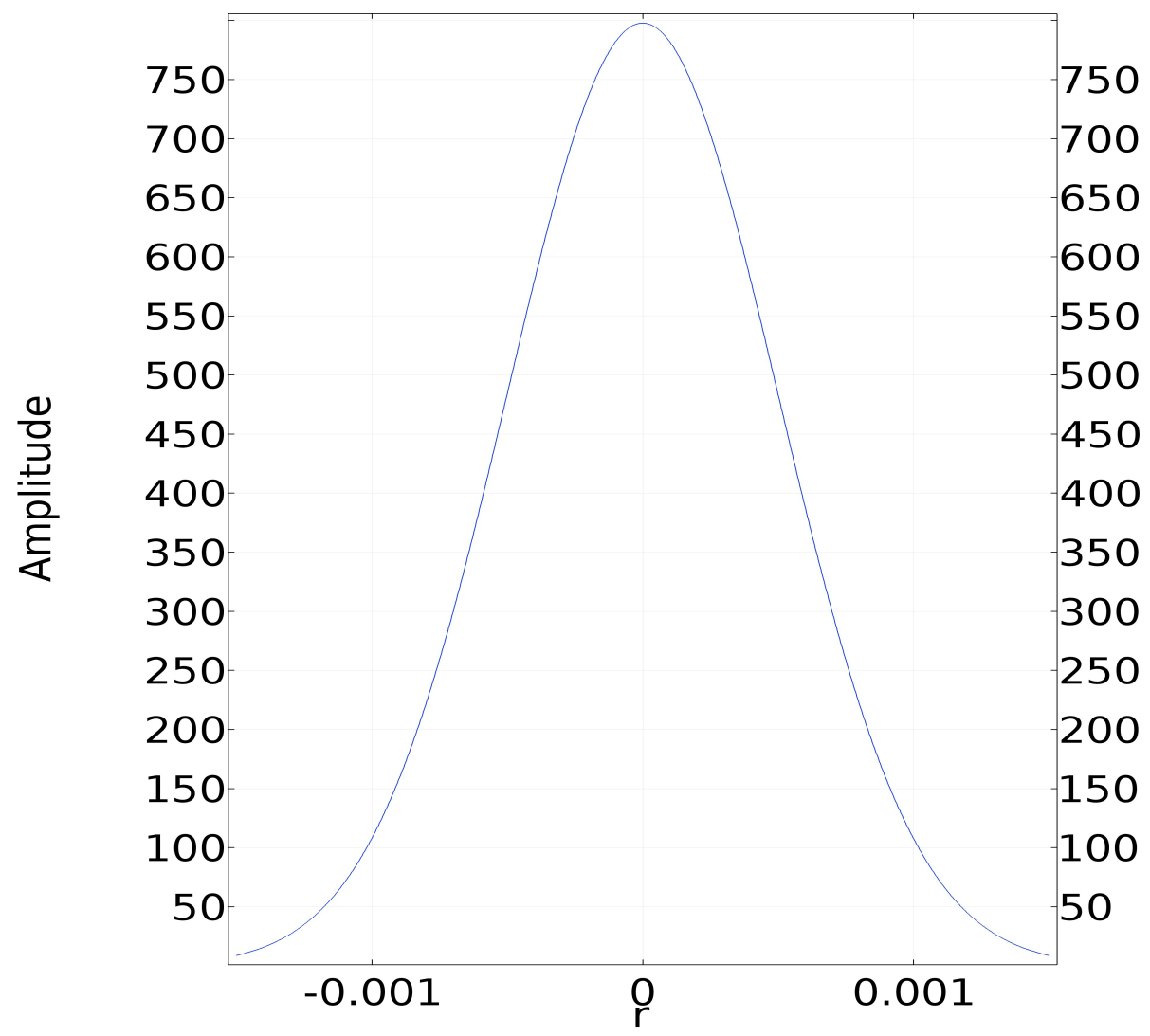

607

Fig. 7

608

609

610

611

612

613

614 
615

616

617

618

619

620

621

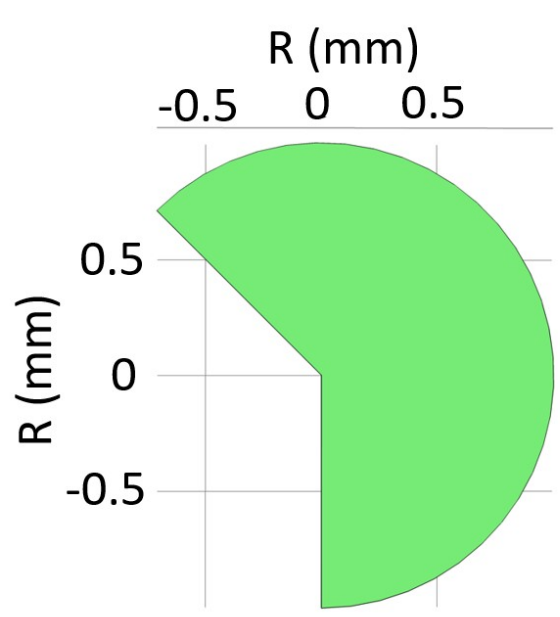

(a)

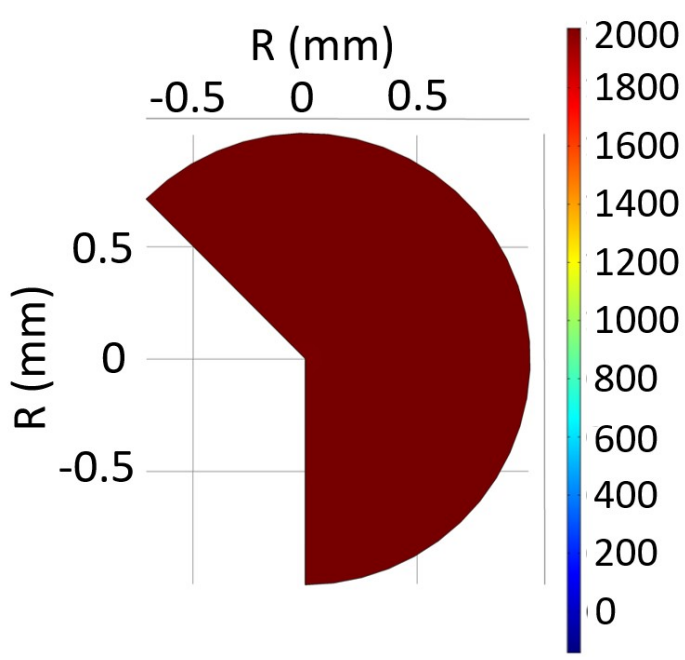

(b)

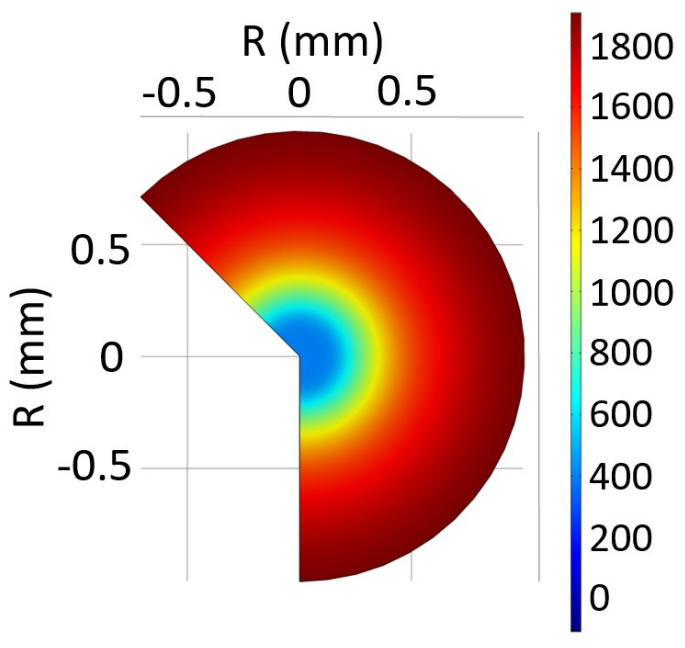

Fig. 8 
622

623

624

625

626

627

628

629

630

631

632

633 (a)
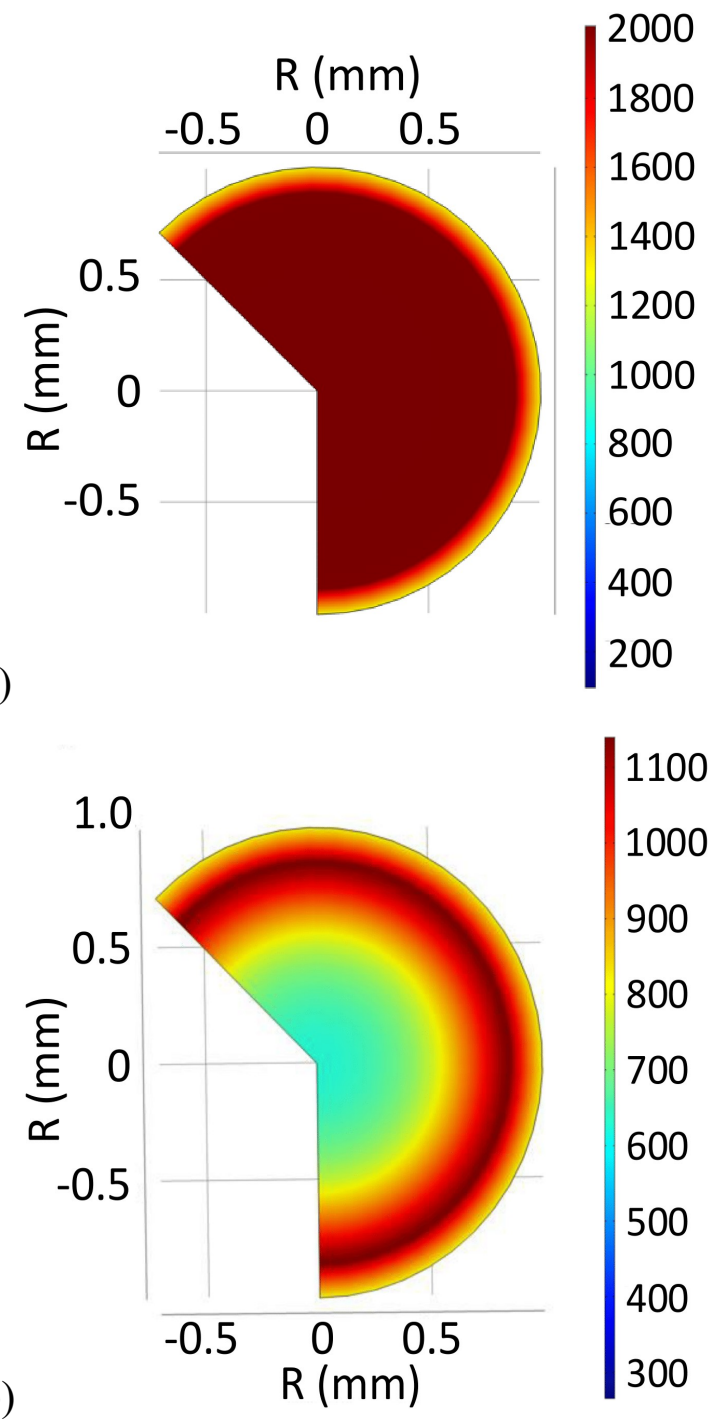

Fig. 9 


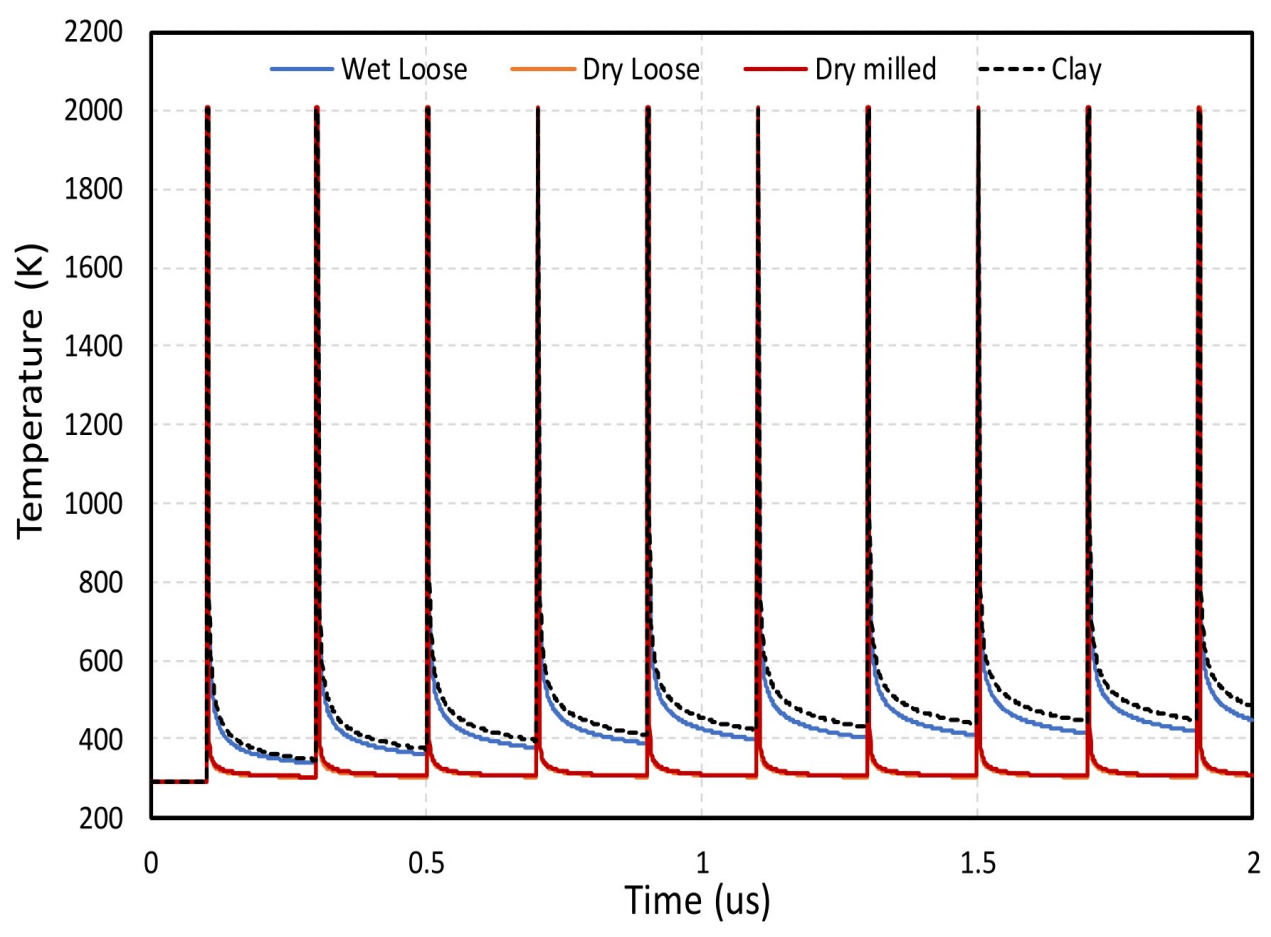

635

636

637

Fig. 10

638

639

640

641

642

643

644

645

646

647

648

649 


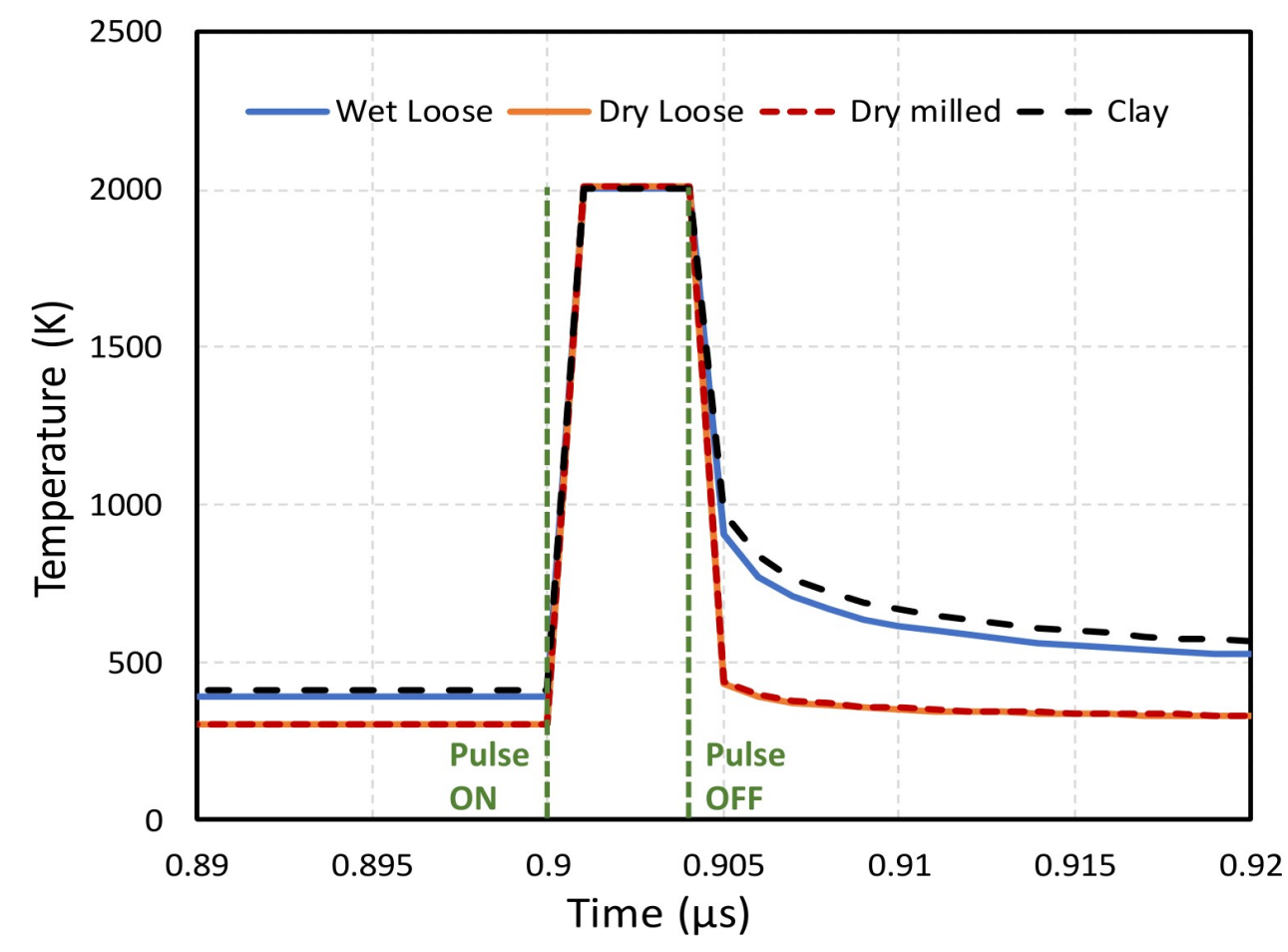

651

652

653

Fig. 11

654

655

656

657

658

659

660

661

662

663

664

665 
666

667

668

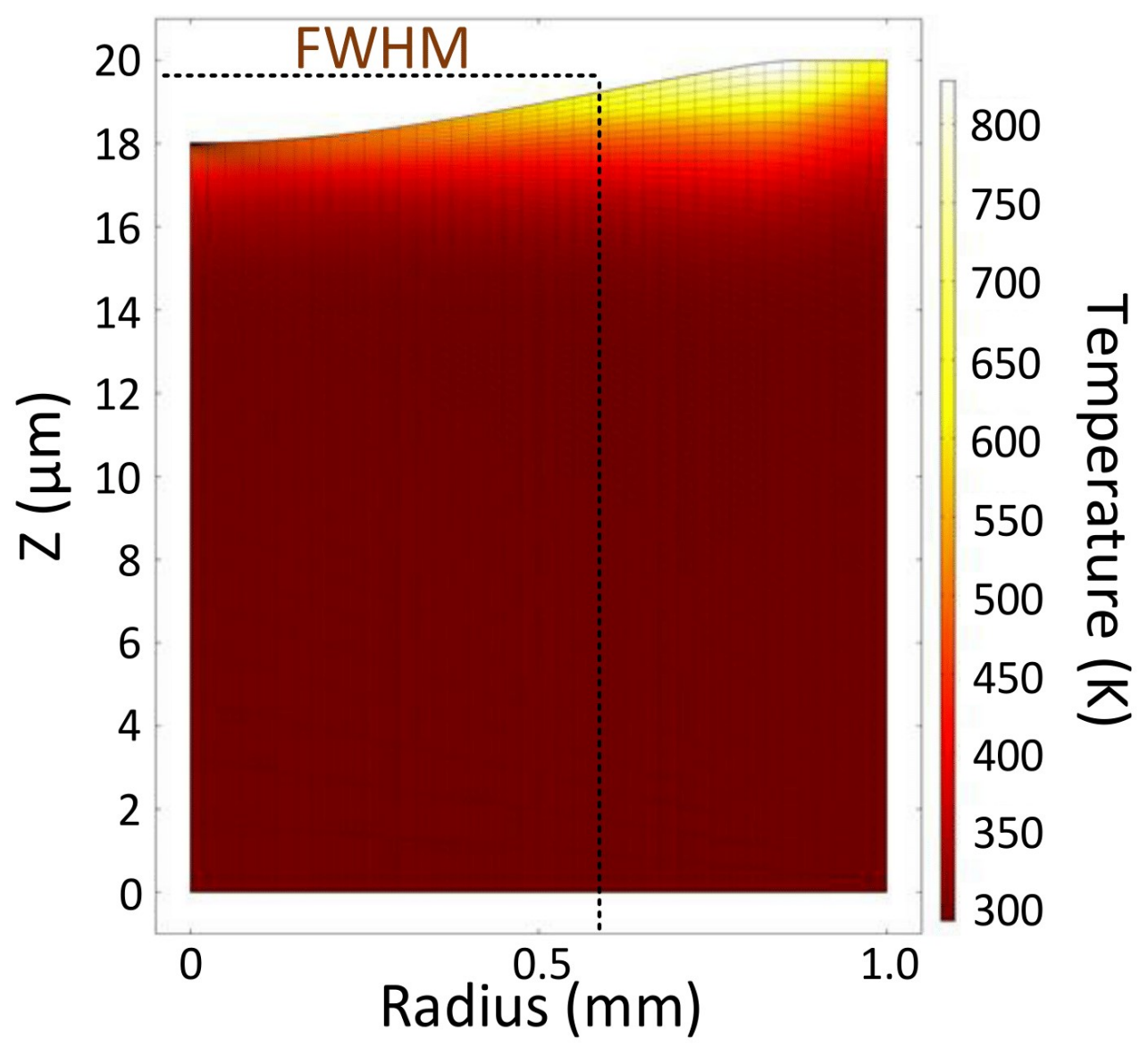

669

670

Fig. 12

671 


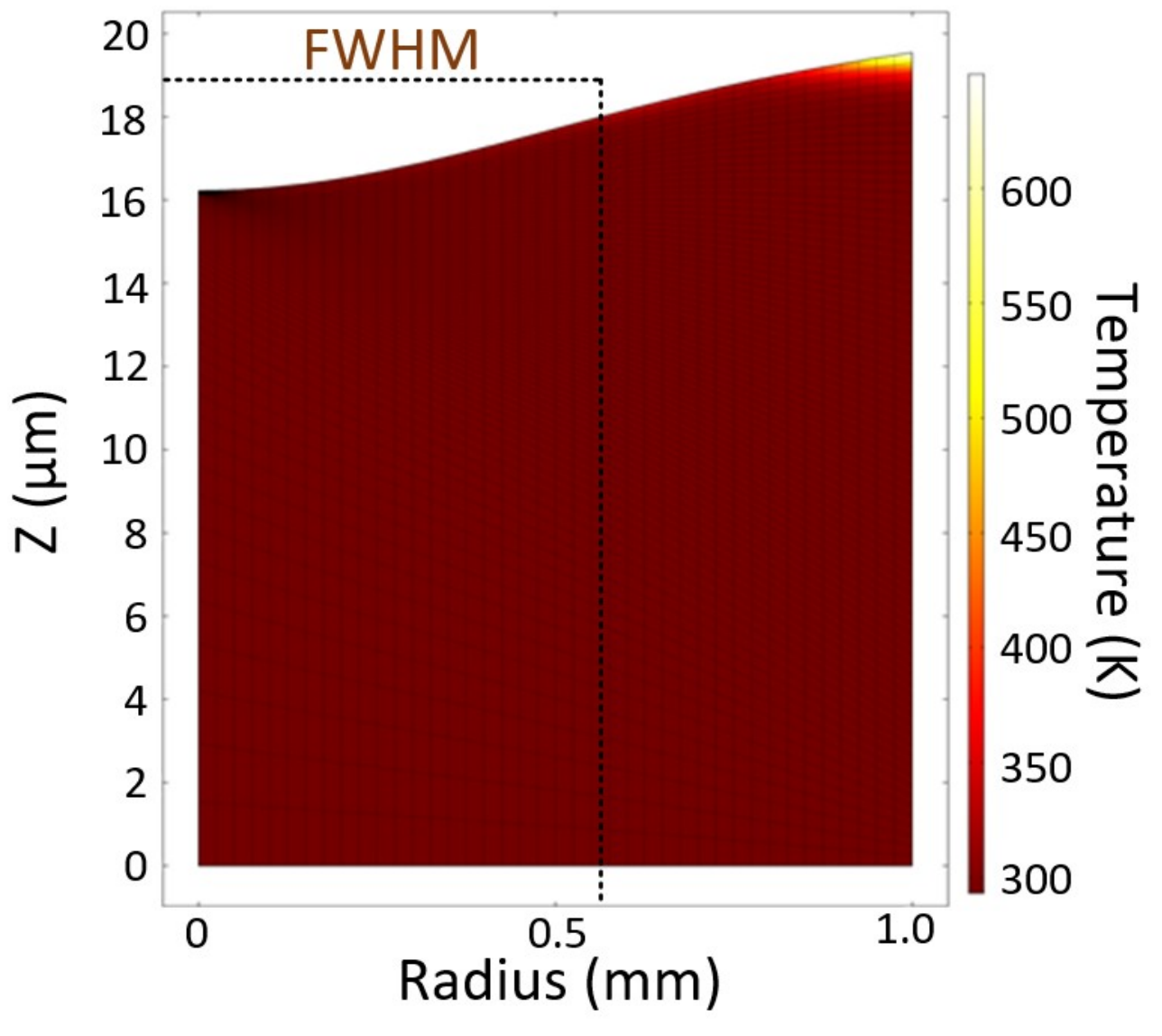

673

674

Fig. 13

675

676

677

678

679

680 


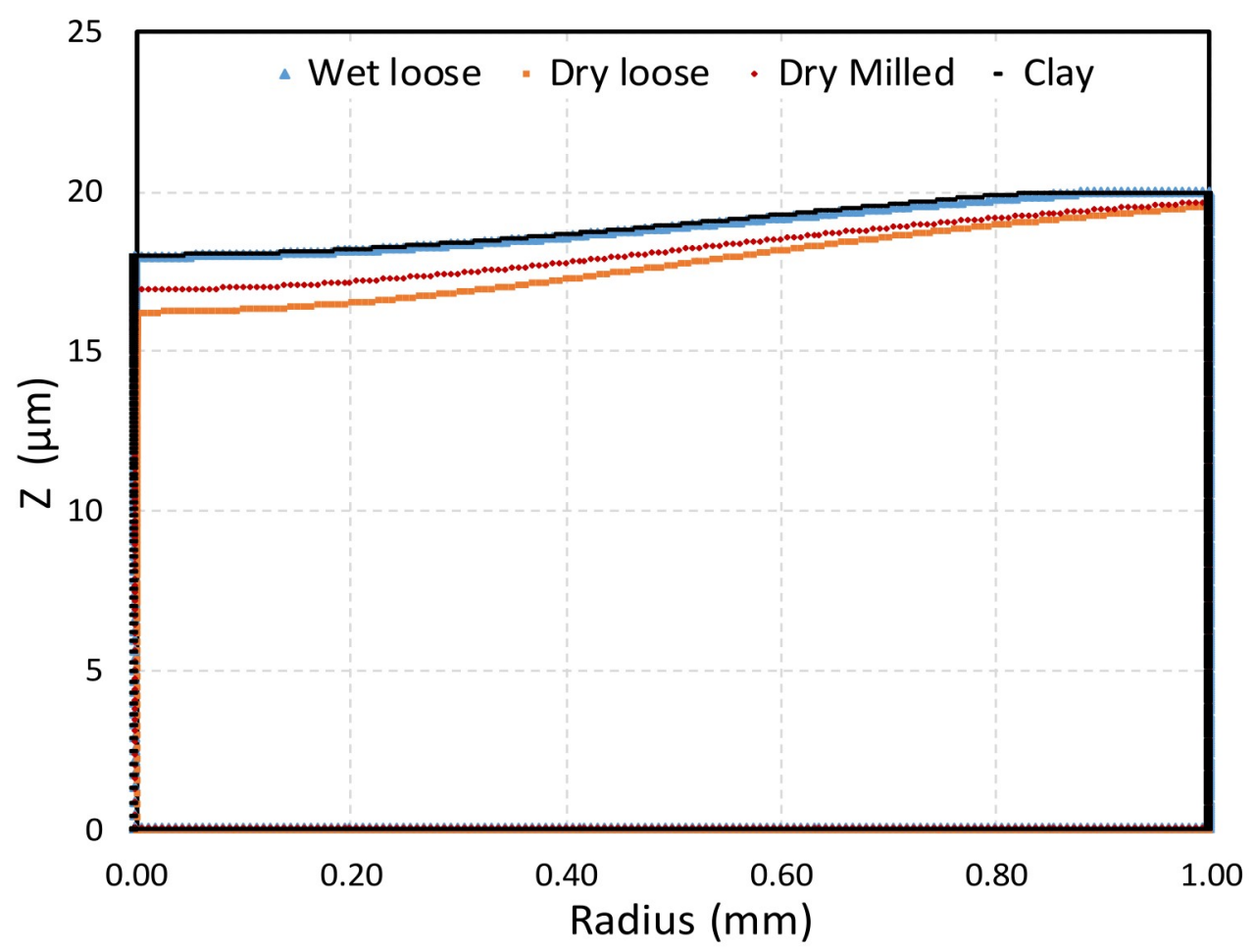

682

683

Fig. 14

684

685

686

687 


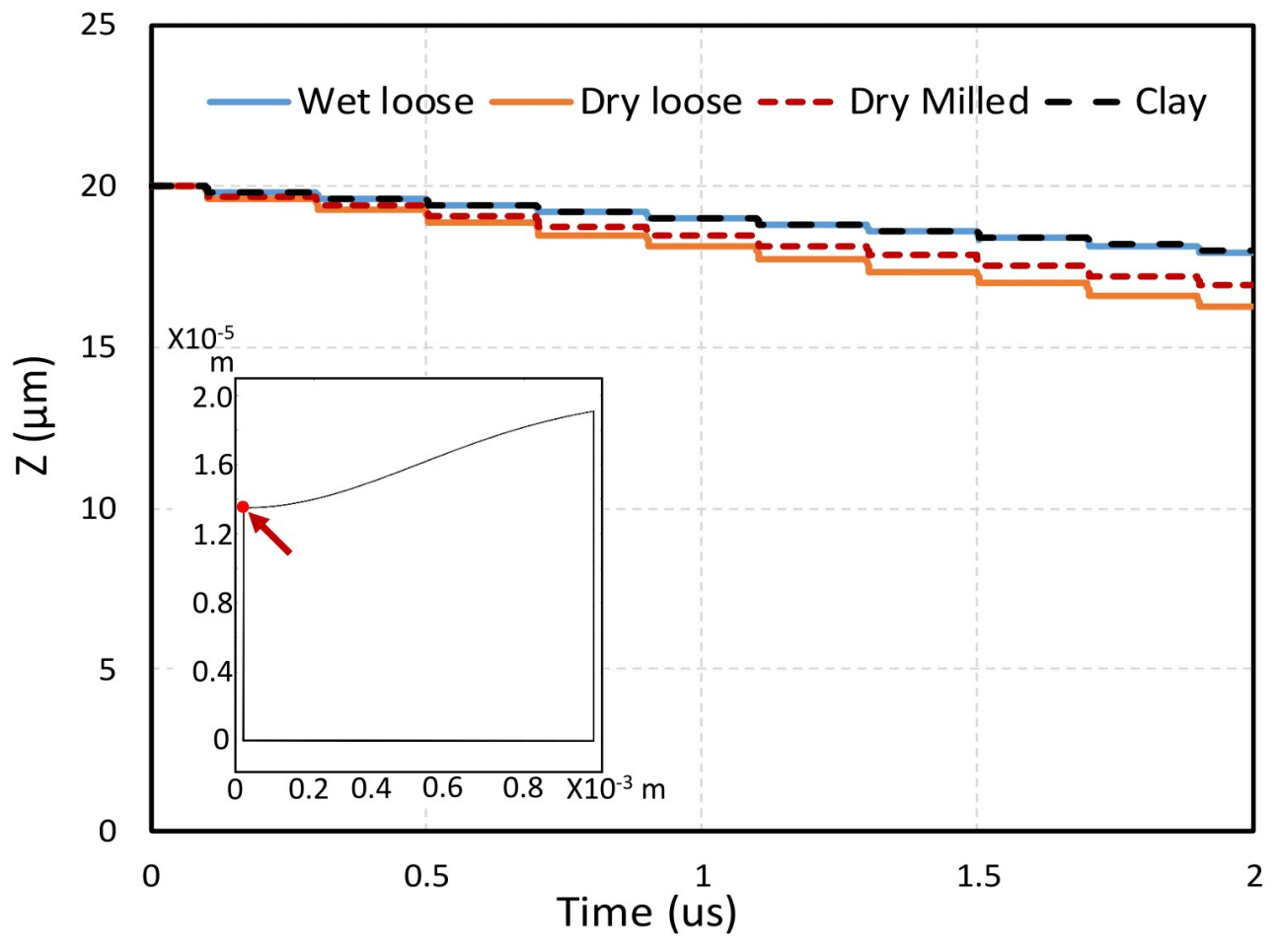

688

689

690

Fig. 15

691

692

693

694

695

696

697

698 


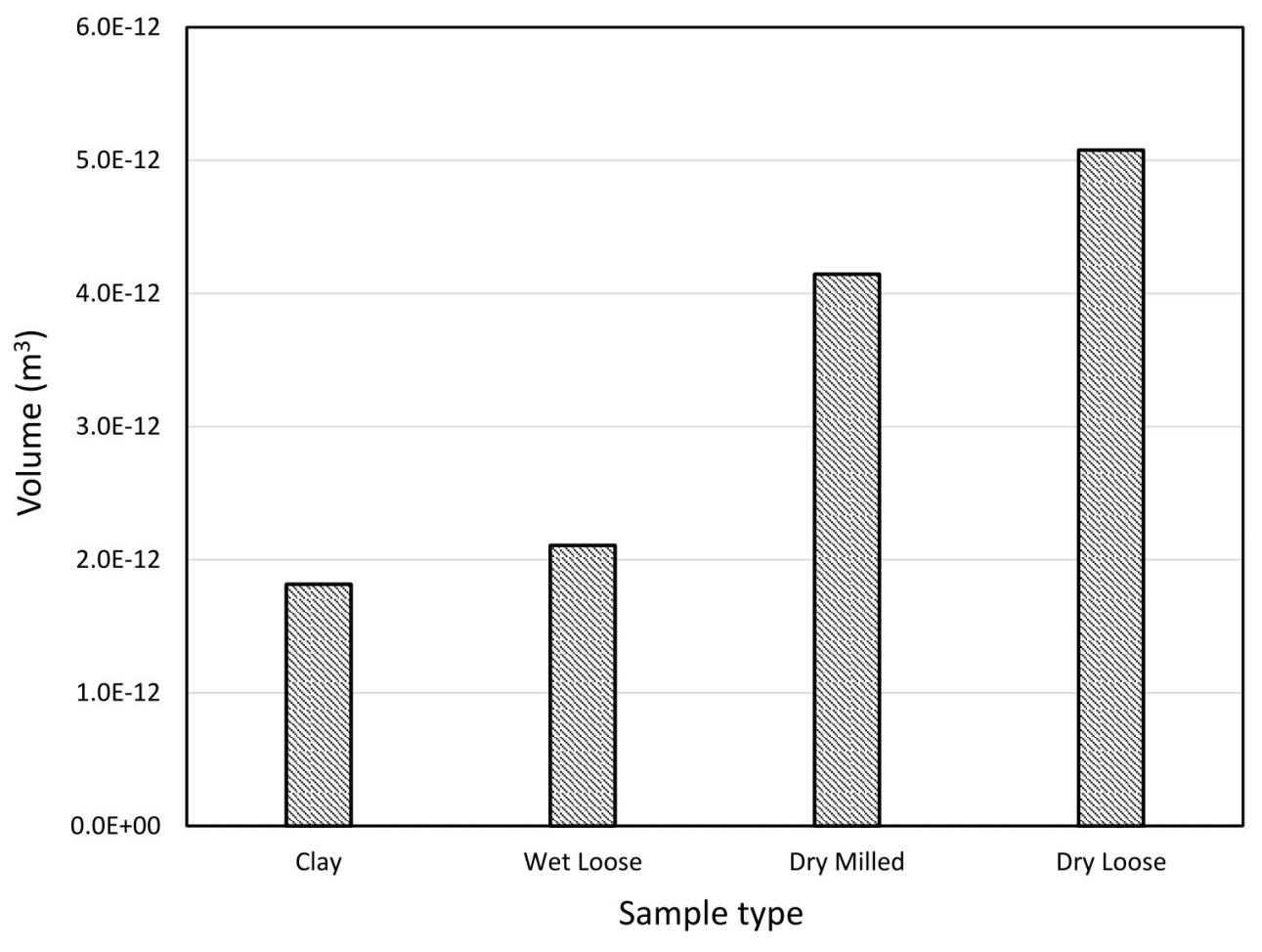

699

700

701

Fig. 16

702

703

704

705

706

707

708

709

710

711

712

713

714 


\section{Tables}

716 TABLE 1

717 SIMULATION PARAMETERS

\begin{tabular}{|c|c|c|}
\hline Property & Nomenclature & Values \\
\hline \multicolumn{3}{|l|}{ Simulation properties } \\
\hline Resolved length scale [m] & $\mathrm{L}_{\text {cond }}$ & $4.33 \times 10^{-8}$ \\
\hline Resolved time $[\mathrm{s}]$ & $t_{\text {step }}$ & $2 \times 10^{-11}$ \\
\hline Ambient temperature $[\mathrm{K}]$ & $\mathrm{T}_{\mathrm{amb}}$ & 293 \\
\hline Mesh resolution (surface) [m] & $\mathrm{h}_{\max }$ & $8.66 \times 10^{-9}$ \\
\hline Maximum simulation time [s] & $\mathrm{T}_{\max }$ & $2 \times 10^{-6}$ \\
\hline \multicolumn{3}{|l|}{ Laser properties } \\
\hline Raw beam diameter $[\mathrm{m}]$ & $2 * r_{\text {spot }}$ & 0.003 \\
\hline Nominal output $[\mathrm{mJ}]$ & Elaser $_{\text {la }}$ & 25 \\
\hline Maximum repetition rate $[\mathrm{Hz}]$ & $\mathrm{f}_{\text {laser }}$ & 1 \\
\hline Nominal pulse width [ns] & & 4 \\
\hline Peak power at $1064 \mathrm{~nm}[\mathrm{MW}]$ & $\mathrm{P}_{\text {laser }}$ & $\sim 2$ \\
\hline Emission Wavelength [nm] & $\lambda_{\text {laser }}$ & 1064 \\
\hline \multicolumn{3}{|l|}{ Laser ablation properties } \\
\hline Number of laser pulses & & 10 \\
\hline Time between pulses $[\mu \mathrm{s}]$ & & 0.196 \\
\hline Pulse width [ns] & & 4 \\
\hline S.D Gaussian laser beam (gp) & $\mathrm{r}_{\mathrm{spot}} / 3$ & 0.001 \\
\hline Ablation temperature $[\mathrm{K}]$ & $\mathrm{T}_{\text {ablate }}$ & 2000 \\
\hline \multicolumn{3}{|l|}{ Sample properties } \\
\hline Initial sample temperature $[\mathrm{K}]$ & $\mathrm{T}_{\text {soil }} / \mathrm{T}_{\text {ext }}$ & 293 \\
\hline \multicolumn{3}{|l|}{ * Soil - dry \& loose } \\
\hline Density $\left[\mathrm{kg} / \mathrm{m}^{3}\right]$ & $\rho_{\text {soil }}$ & 1600 \\
\hline Moisture content [\%] & $\mathrm{W}_{\text {soil }}$ & 0 \\
\hline Porosity $[\%]$ & $\varepsilon$ & 46 \\
\hline Thermal conductivity $[\mathrm{W} / \mathrm{mK}]$ & $\mathrm{k}_{\text {soil }}$ & 0.15 \\
\hline Specific heat capacity [J/kg K] & $\mathrm{Cp}_{\text {soil }}$ & 800 \\
\hline \multicolumn{3}{|l|}{ * Soil - dry \& compressed } \\
\hline Density $\left[\mathrm{kg} / \mathrm{m}^{3}\right]$ & $\rho_{\text {soil }}$ & 1950 \\
\hline Moisture content [\%] & $\mathrm{W}_{\text {soil }}$ & 0 \\
\hline Porosity $[\%]$ & $\varepsilon$ & 29 \\
\hline Thermal conductivity $[\mathrm{W} / \mathrm{mK}]$ & $\mathrm{k}_{\text {soil }}$ & 0.15 \\
\hline Specific heat capacity [J/kg K] & $\mathrm{Cp}_{\text {soil }}$ & 800 \\
\hline \multicolumn{3}{|l|}{ * Soil - wet \& loose } \\
\hline Density $\left[\mathrm{kg} / \mathrm{m}^{3}\right]$ & $\rho_{\text {soil }}$ & 1613 \\
\hline
\end{tabular}




\begin{tabular}{|l|c|c|}
\hline Moisture content [\%] & $\mathrm{W}_{\text {soil }}$ & 24.6 \\
\hline Porosity [\%] & $\varepsilon$ & 39 \\
\hline Thermal conductivity [W/mK] & $\mathrm{k}_{\text {soil }}$ & 2.75 \\
\hline Specific heat capacity [J/kg K] & $\mathrm{Cp}_{\text {soil }}$ & 1632 \\
\hline & & \\
\hline * Soil - fine sandy clay & & \\
\hline Density [kg/m $\left.{ }^{3}\right]$ & & 1067 \\
\hline Moisture content [\%] & $\rho_{\text {soil }}$ & 54.6 \\
\hline Porosity [\%] & $\mathrm{W}_{\text {soil }}$ & 37 \\
\hline Thermal conductivity [W/mK] & $\varepsilon$ & 4.20 \\
\hline Specific heat capacity [J/kg K] & $\mathrm{k}_{\text {soil }}$ & 2646 \\
\hline
\end{tabular}

TABLE 2

BOUNDARY CONDITION EQUATIONS

\begin{tabular}{|c|c|c|}
\hline Boundary No. & Boundary condition & Equation \\
\hline \multirow[t]{6}{*}{ Whole geometry } & Heat transfer coefficient & $q=-k \nabla T$ \\
\hline & $\begin{array}{l}\text { General heat equation (where, } Q \\
\text { is heat source) }\end{array}$ & $\rho C_{p} \frac{\partial T}{\partial t}=\nabla \cdot(k \nabla T)+Q$ \\
\hline & Conduction length & $2 * \operatorname{sqrt}\left(\alpha_{\text {cond }} * t_{\text {step }}\right)$ \\
\hline & Soil thermal diffusivity $\left(\alpha_{\text {cond }}\right)$ & $\mathrm{k}_{\text {soil }} / \mathrm{C} p_{\text {soil }} / \rho_{\text {soil }}$ \\
\hline & Resolved length scale & $6 * \operatorname{sqrt}\left(\alpha_{\text {cond }} * t_{\max }\right)$ \\
\hline & Conduction length & $2 * \operatorname{sqrt}\left(\alpha_{\text {cond }} * t_{\text {step }}\right)$ \\
\hline 1 & $\begin{array}{l}\text { Laser power (Gaussian } \\
\text { distribution (gp)) }\end{array}$ & $\begin{array}{l}\mathrm{P}_{\text {laser }}\left[\mathrm{kW} / \mathrm{m}^{2}\right]^{*}(\mathrm{gp}(\mathrm{x}) / 800)^{*} \\
\text { pulse }(\mathrm{t})\end{array}$ \\
\hline 1,4 & Ablation velocity & $\begin{array}{l}\text { ht.P } P_{\text {laser. }} \mathrm{q} 0 /\left(\rho_{\text {soil }}\left[\mathrm{kg} / \mathrm{m}^{3}\right]^{*} \mathrm{H}_{\mathrm{fs}}\right. \\
[\mathrm{kJ} / \mathrm{kg}])\end{array}$ \\
\hline 2,3 & $\begin{array}{l}\text { Thermal insulation (where, } n \text { is } \\
\text { refractive index) }\end{array}$ & $-n \cdot q=0$ \\
\hline 1 & Heat flux (ht) & $-n \cdot q=q_{0}$ \\
\hline 1 & Convective heat flux & $\mathrm{q}_{0}=\mathrm{h} \bullet\left(\mathrm{T}_{\mathrm{ext}}-\mathrm{T}\right)$ \\
\hline 1 & Heat transfer coefficient (ht) & $\left.\left(\mathrm{T}-\mathrm{T}_{\text {ablate }}\right)[1 / \mathrm{K}]\right)$ \\
\hline 1 & Diffusive surface & -n.q $=\varepsilon \sigma\left(T^{4}{ }_{a m b}-T^{4}\right)$ \\
\hline 4 & $\mathrm{~h}_{\max }$ & Conduction length/5 \\
\hline $1,2,3$ & $\mathrm{~L}_{\text {cond }}$ & $2 * \operatorname{sqrt}\left(\alpha_{\text {cond }} * t_{\text {step }}\right)$ \\
\hline
\end{tabular}


727 


\section{References}

729 Andrew Knight et al, "Characterization of Laser-Induced Breakdown

730 Spectroscopy (LIBS) for Application to Space Exploration", Applied

731 Spectroscopy, Vol 54, No. 3, 2000.

732

733 Bruno Bousquet et al, "Towards quantitative laser-induced breakdown

734 spectroscopy analysis of soil samples", Spectrochimica Acta Part B, 62, 1582$7351589,2007$.

736

737 Gabriel Gustinelli Arantes de Carvalho et al, Influence of particle size 738 distribution on the analysis of pellets of plant materials by laser-induced 739 breakdown spectroscopy, Spectrochim. Acta Part B, 2015, 105, 130-135.

741 David Hahn and Nicolo. Omenetto. "Laser-Induced Breakdown Spectroscopy

742 (LIBS), Part II: Review of Instrumental and Methodological Approaches to

743 Material Analysis and Applications to Different Fields”, Applied Spectroscopy 744 Vol 66, No.4, 2012.

745

746 Fabio Castell et al, "Estimation of surface heat flux and an index of soil moisture

747 using adjoint-state surface energy balance", Water resources research, 35, 10, $748 \quad 3115-3125,1999$.

749

750 Francesco Capitelli et al, "Determination of heavy metals in soils by Laser

751 Induced Breakdown spectroscopy”, Geoderma, 106, 45-62, 2002. 
752 Francisco Anabitarte et al. "Laser-Induced Breakdown Spectroscopy:

753 Fundamentals, Applications, and Challenges", International Scholarly Research

754 Network ISRN Spectroscopy, Article ID 285240, 12 pages, 2012.

755

756 Gabriel. Gustinelli et al. "Influence of particle size distribution on the analysis of 757 pellets of plant materials by laser-induced breakdown spectroscopy", 758 Spectrochimica Acta, Part B 105, 130-135, 2015.

759

760 Ines. Rauschenbach et al, "Laser induced breakdown spectroscopy on soils and

761 rocks: Influence of the sample temperature, moisture and roughness", 762 Spectrochimica Acta, Part B 63, 1205-1215, 2008.

763

764 Jiangbo Han et al, "Dynamics of soil water evaporation during soil drying:

765 Laboratory experiment and numerical analysis”, Scientific World Journal, 2013.

766

767 Jiyu Peng, "Challenging applications for multi-element analysis by laser-induced 768 breakdown spectroscopy in agriculture: A Review", Trends in Analytical 769 chemistry, 85, 260-272, 2016.

770

771 Junsei Kondo, Nobuko Saigusa, "Modelling the evaporation from bare soil with 772 a formula for vaporization in the soil pores", Journal of the Meterological 773 Society of Japan, 72, 3, 1994. 
775 Justin Freeman et al, "Comparison of optical emission from ns and femtosecond

776 laser produced plasma in atmosphere and vacuum conditions." Spectrochimica

$777 \quad$ Acta Part B 87, 43-50, 2013.

778

779 Lakesh Sharma, Sukhwinder Bali, “A Review of Methods to Improve Nitrogen

780 Use Efficiency in Agriculture”, MDPI Sustainability, 10, 51, 2018.

781

782 Leon. Radziemski, David Cremers. "A brief history of laser-induced breakdown

783 spectroscopy: From the concept of atoms to LIBS 2012", Spectrochimica Acta

784 Part B 87, 3-10, 2013.

785

786 Lilian Trevizan et al. "Evaluation of laser induced breakdown spectroscopy for

787 the determination of macronutrients in plant materials", Spectrochimica Acta 788 Part B 63, 1151-1158, 2008.

789

790 Mark Post et al, "Autonomous Navigation with ROS for a Mobile Robot in

791 Agricultural Fields", 14th International Conference on Informatics in Control,

792 Automation and Robotics (ICINCO), 2017.

793

794 Ningfang Yang. "Elemental Analysis of Soils Using Laser-Induced Breakdown

795 Spectroscopy (LIBS)". The University of Tennessee, Knoxville. Master of

796 science thesis, August 2009.

797

798 Rosalba Gaudiuso et al, "Laser Induced Breakdown Spectroscopy for Elemental

799 Analysis in Environmental, Cultural Heritage and Space, 2010. 
800 Russell Harmon et al (a), "Laser-induced breakdown spectroscopy (LIBS) - an

801 emerging field-portable sensor technology for real-time, in-situ geochemical and

802 environmental analysis", Geochemistry: Exploration, Environment, Analysis,

803 Vol. 5, pp. 21-28, 2005.

804

805 Russell Harmon et al (b) "Applications of laser-induced breakdown spectroscopy

806 for geochemical and environmental analysis: A comprehensive review",

807 Spectrochimica Acta, Part B 87, 11-26, 2013.

808

809 Sarah Jantzi and Jose Almirall, "Characterization and forensic analysis of soil

810 samples using laser-induced breakdown spectroscopy (LIBS)”, Anal Bioanal

811 Chem, 400, 3341-3351, 2011.

812

813 Talib. Hussain and Mohammed Gondal. "Laser induced breakdown spectroscopy

814 (LIBS) as a rapid tool for material analysis", 6th Vacuum and Surface Sciences

815 Conference of Asia and Australia (VASSCAA-6). Journal of Physics:

816 Conference Series 439, 012050, 2013.

817

818 Thomas Sauer, "Soil heat flux”, Nebraska, USDA-ARS/UNL Faculty, 2005.

819

820 Violeta Lazic et al, "Laser induced breakdown spectroscopy of soils, rocks and

821 ice at subzero temperatures in simulated martian conditions", Spectrochimica 822 Acta, Part B 62, 1546 - 1556, 2007.

823 
824 Yong He et al, "Quantitative Analysis of Nutrient Elements in Soil Using Single 825 and Double-Pulse Laser-Induced Breakdown Spectroscopy”, MDPI Sensors, 18, $8261526,2018$. 